\title{
The Over-Relaxed $A$-Proximal Point Algorithm for General Nonlinear Mixed Set-Valued Inclusion Framework
}

\author{
Xian Bing Pan, ${ }^{1}$ Hong Gang $\mathrm{Li}^{2}{ }^{2}$ and An Jian $X u^{3}$ \\ ${ }^{1}$ Yitong College, Chongqing University of Posts and Telecommunications, Chongqing 400065, China \\ 2 Institute of Applied Mathematics Research, Chongqing University of Posts and Telecommunications, \\ Chongqing 400065, China \\ ${ }^{3}$ College of Mathematics and Statistics, Chongqing University of Technology, Chongqing 400054, China
}

Correspondence should be addressed to Xian Bing Pan, panxianb@163.com

Received 16 November 2010; Accepted 10 January 2011

Academic Editor: T. Benavides

Copyright (C 2011 Xian Bing Pan et al. This is an open access article distributed under the Creative Commons Attribution License, which permits unrestricted use, distribution, and reproduction in any medium, provided the original work is properly cited.

The purpose of this paper is (1) a general nonlinear mixed set-valued inclusion framework for the over-relaxed $A$-proximal point algorithm based on the $(A, \eta)$-accretive mapping is introduced, and (2) it is applied to the approximation solvability of a general class of inclusions problems using the generalized resolvent operator technique due to Lan-Cho-Verma, and the convergence of iterative sequences generated by the algorithm is discussed in $q$-uniformly smooth Banach spaces. The results presented in the paper improve and extend some known results in the literature.

\section{Introduction}

In recent years, various set-valued variational inclusion frameworks, which have wide applications to many fields including, for example, mechanics, physics, optimization and control, nonlinear programming, economics, and engineering sciences have been intensively studied by Ding and Luo [1], Verma [2], Huang [3], Fang and Huang [4], Fang et al. [5], Lan et al. [6], Zhang et al. [7], respectively. Recently, Verma [8] has intended to develop a general inclusion framework for the over-relaxed $A$-proximal point algorithm [9] based on the A-maximal monotonicity. In 2007-2008, Li $[10,11]$ has studied the algorithm for a new class of generalized nonlinear fuzzy set-valued variational inclusions involving $(H, \eta)$ monotone mappings and an existence theorem of solutions for the variational inclusions, and a new iterative algorithm [12] for a new class of general nonlinear fuzzy mulitvalued quasivariational inclusions involving $(G, \eta)$-monotone mappings in Hilbert spaces, and discussed a new perturbed Ishikawa iterative algorithm for nonlinear mixed set-valued 
quasivariational inclusions involving $(A, \eta)$-accretive mappings, the stability [13] and the convergence of the iterative sequences in $q$-uniformly smooth Banach spaces by using the resolvent operator technique due to Lan et al. [6].

Inspired and motivated by recent research work in this field, in this paper, a general nonlinear mixed set-valued inclusion framework for the over-relaxed $A$-proximal point algorithm based on the $(A, \eta)$-accretive mapping is introduced, which is applied to the approximation solvability of a general class of inclusions problems by the generalized resolvent operator technique, and the convergence of iterative sequences generated by the algorithm is discussed in $q$-uniformly smooth Banach spaces. For more literature, we recommend to the reader [1-17].

\section{Preliminaries}

Let $X$ be a real Banach space with dual space $X^{*}$, and let $\langle\cdot, \cdot\rangle$ be the dual pair between $X$ and $X^{*}$, let $2^{X}$ denote the family of all the nonempty subsets of $X$, and let $\mathrm{CB}(X)$ denote the family of all nonempty closed bounded subsets of $X$. The generalized duality mapping $J_{q}: X \rightarrow 2^{X^{*}}$ is single-valued if $X^{*}$ is strictly convex [14], or $X$ is uniformly smooth space. In what follows we always denote the single-valued generalized duality mapping by $J_{q}$ in real uniformly smooth Banach space $X$ unless otherwise stated. We consider the following general nonlinear mixed set-valued inclusion problem with $(A, \eta)$-accretive mappings (GNMSVIP).

Finding $x \in X$ such that

$$
0 \in F(A(x))+M(x)
$$

where $A, F: X \rightarrow X, \eta: X \times X \rightarrow X$ be single-valued mappings; $M: X \rightarrow 2^{X}$ be an $(A, \eta)$-accretive set-valued mapping. A special case of problem (2.1) is the following:

if $X=X^{*}$ is a Hilbert space, $F=0$ is the zero operator in $X$, and $\eta(x, y)=x-y$, then problem (2.1) becomes the inclusion problem $0 \in M(x)$ with a $A$-maximal monotone mapping $M$, which was studied by Verma [8].

Definition 2.1. Let $X$ be a real Banach space with dual space $X^{*}$, and let $\langle\cdot, \cdot\rangle$ be the dual pair between $X$ and $X^{*}$. Let $A: X \rightarrow X$ and $\eta: X \times X \rightarrow X$ be single-valued mappings. A set-valued mapping $M: X \rightarrow 2^{X}$ is said to be

(i) $r$-strongly $\eta$-accretive, if there exists a constant $r>0$ such that

$$
\left\langle y_{1}-y_{2}, J_{q}\left(\eta\left(x_{1}, x_{2}\right)\right)\right\rangle \geq r\left\|x_{1}-x_{2}\right\|^{q}, \quad \forall y_{i} \in M\left(x_{i}\right), i=1,2 ;
$$

(ii) $m$-relaxed $\eta$-accretive, if there exists a constant $m>0$ such that

$\left\langle y_{1}-y_{2}, J_{q}\left(\eta\left(x_{1}, x_{2}\right)\right)\right\rangle \geq-m\left\|x_{1}-x_{2}\right\|^{q}, \quad \forall x_{1}, x_{2} \in X, y_{i} \in M\left(x_{i}\right), \quad(i=1,2) ;$

(iii) $c$-cocoercive, if there exists a constant $c$ such that

$$
\left\langle y_{1}-y_{2}, J_{q}\left(\eta\left(x_{1}, x_{2}\right)\right)\right\rangle \geq c\left\|y_{1}-y_{2}\right\|^{q}, \quad \forall x_{1}, x_{2} \in X, y_{i} \in M\left(x_{i}\right), \quad(i=1,2) ;
$$

(iv) $(A, \eta)$-accretive, if $M$ is $m$-relaxed $\eta$-accretive and $R(A+\rho M)(X)=X$ for every $\rho>0$. 
Based on the literature [6], we can define the resolvent operator $R_{\rho, M}^{A, \eta}$ as follows.

Definition 2.2. Let $\eta: X \times X \rightarrow X$ be a single-valued mapping, $A: X \rightarrow X$ be a strictly $\eta$-accretive single-valued mapping and $M: X \rightarrow 2^{X}$ be an $(A, \eta)$-accretive set-valued mapping. The resolvent operator $R_{\rho, M}^{A, \eta}: X \rightarrow X$ is defined by

$$
R_{\rho, M}^{A, \eta}(x)=(A+\rho M)^{-1}(x) \quad(\forall x \in X),
$$

where $\rho>0$ is a constant.

Remark 2.3. The $(A, \eta)$-accretive mappings are more general than $(H, \eta)$-monotone mappings and $m$-accretive mappings in Banach space or Hilbert space, and the resolvent operators associated with $(A, \eta)$-accretive mappings include as special cases the corresponding resolvent operators associated with $(H, \eta)$-monotone operators, $m$-accretive mappings, $A$ monotone operators, $\eta$-subdifferential operators $[1-7,11-13]$.

Lemma 2.4 (see [6]). Let $\eta: X \times X \rightarrow X$ be $\tau$-Lipschtiz continuous mapping, $A: X \rightarrow X$ be an $r$-strongly $\eta$-accretive mapping, and $M: X \rightarrow 2^{X}$ be an $(A, \eta)$-accretive set-valued mapping. Then the generalized resolvent operator $R_{\rho, M}^{A, \eta}: X \rightarrow X$ is $\tau^{q-1} /(r-m \rho)$-Lipschitz continuous, that is,

$$
\left\|R_{\rho, M}^{A, \eta}(x)-R_{\rho, M}^{A, \eta}(y)\right\| \leq \frac{\tau^{q-1}}{r-m \rho}\|x-y\| \quad(\forall x, y \in X),
$$

where $\rho \in(0, r / m)$.

In the study of characteristic inequalities in $q$-uniformly smooth Banach spaces, $\mathrm{Xu}$ [14] proved the following result.

Lemma 2.5. Let $X$ be a real uniformly smooth Banach space. Then $X$ is q-uniformly smooth if and only if there exists a constant $c_{q}>0$ such that for all $x, y \in X$,

$$
\|x+y\|^{q} \leq\|x\|^{q}+q\left\langle y, J_{q}(x)\right\rangle+c_{q}\|y\|^{q} .
$$

\section{The Over-Relaxed $A$-Proximal Point Algorithm}

This section deals with an introduction of a generalized version of the over-relaxed proximal point algorithm and its applications to approximation solvability of the inclusion problem of the form $(2.1)$ based on the $(A, \eta)$-accretive set-valued mapping.

Let $M: X \rightarrow 2^{X}$ be a set-valued mapping, the set $\{(x, y): y \in M(x)\}$ be the graph of $M$, which is denoted by $M$ for simplicity, This is equivalent to stating that a mapping is any subset $M$ of $X \times X$, and $M(x)=\{y:(x, y) \in M\}$. If $M$ is single-valued, we shall still use 
$M(x)$ to represent the unique y such that $(x, y) \in M$ rather than the singleton set $\{y\}$. This interpretation will depend greatly on the context. The inverse $M^{-1}$ of $M$ is $\{(y, x):(x, y) \in$ $M\}$.

Definition 3.1. Let $M: X \rightarrow 2^{X}$ be a set-valued mapping. The map $M^{-1}$, the inverse of $M: X \rightarrow 2^{X}$, is said to be general $(u, t)$-Lipschitz continuous at 0 if, and only if there exist two constants $u, t \geq 0$ for any $w \in B_{t}=\{w:\|w\| \leq t, w \in X\}$, a solution $x^{*}$ of the inclusion $0 \in M(x)\left(x^{*} \in M^{-1}(0)\right)$ exist and the $x^{*}$ such that

$$
\left\|x-x^{*}\right\| \leq u\|w\| \quad\left(\forall x \in M^{-1}(w)\right)
$$

holds.

Lemma 3.2. Let $X$ be a q-uniformly smooth Banach space, $\eta: X \times X \rightarrow X$ be a $\tau$-Lipschtiz continuous mapping, $A: X \rightarrow X$ be an r-strongly $\eta$-accretive mapping, $F: X \rightarrow X$ be a $\xi$ Lipschtiz continuous mapping, and $M: X \rightarrow 2^{X}$ be an $(A, \eta)$-accretive set-valued mapping. If $I_{k}=A-A R_{\rho, M}^{A, \eta}(A-\rho F A)$, and for all $x_{1}, x_{2} \in X, \rho>0$ and $q \gamma>1$

$$
\begin{gathered}
\left\langle A\left(x_{1}\right)-A\left(x_{2}\right), J_{q}\left(A\left(R_{\rho, M}^{A, \eta}\left(A\left(x_{1}\right)-\rho F\left(A\left(x_{1}\right)\right)\right)\right)-A\left(R_{\rho, M}^{A, \eta}\left(A\left(x_{2}\right)-\rho F\left(A\left(x_{2}\right)\right)\right)\right)\right)\right\rangle \\
\geq r\left\|A\left(R_{\rho, M}^{A, \eta}\left(A\left(x_{1}\right)-\rho F\left(A\left(x_{1}\right)\right)\right)\right)-A\left(R_{\rho, M}^{A, \eta}\left(A\left(x_{2}\right)-\rho F\left(A\left(x_{2}\right)\right)\right)\right)\right\|^{q},
\end{gathered}
$$

then

$$
\begin{gathered}
(q r-1)\left\|A\left(R_{\rho, M}^{A, \eta}\left(A\left(x_{1}\right)-\rho F\left(A\left(x_{1}\right)\right)\right)\right)-A\left(R_{\rho, M}^{A, \eta}\left(A\left(x_{2}\right)-\rho F\left(A\left(x_{2}\right)\right)\right)\right)\right\|^{q} \\
+\left\|I_{k}\left(x_{1}\right)-I_{k}\left(x_{2}\right)\right\|^{q} \leq c_{q}\left\|A\left(x_{1}\right)-A\left(x_{2}\right)\right\|^{q} .
\end{gathered}
$$

Proof. Let $X$ be a $q$-uniformly smooth Banach space, $\eta: X \times X \rightarrow X$ be a $\tau$-Lipschtiz continuous mapping, $A: X \rightarrow X$ be an $r$-strongly $\eta$-accretive mapping, and $M: X \rightarrow 2^{X}$ be an $(A, \eta)$-accretive set-valued mapping. Let us set $I_{k}=A-A R_{\rho, M}^{A, \eta}(A-\rho F A)$ and $s_{i}=A\left(x_{i}\right)-\rho F\left(A\left(x_{i}\right)\right)\left(x_{i} \in X, i=1,2\right)$, then by using Definition 2.2, Lemmas 2.4, 2.5, and (3.2), we can have

$$
\begin{aligned}
& \left\|I_{k}\left(x_{1}\right)-I_{k}\left(x_{2}\right)\right\|^{q} \\
& \quad=\left\|A\left(x_{1}\right)-A\left(R_{\rho, M}^{A, \eta}\left(s_{1}\right)\right)-\left[A\left(x_{2}\right)-A\left(R_{\rho, M}^{A, \eta}\left(s_{2}\right)\right)\right]\right\|^{q}
\end{aligned}
$$


Fixed Point Theory and Applications

$$
\begin{aligned}
\leq & c_{q}\left\|A\left(x_{1}\right)-A\left(x_{2}\right)\right\|^{q}-q\left\langle A\left(x_{1}\right)-A\left(x_{2}\right), J_{q}\left(A\left(R_{\rho, M}^{A, \eta}\left(s_{1}\right)\right)-A\left(R_{\rho, M}^{A, \eta}\left(s_{2}\right)\right)\right)\right\rangle \\
& +\left\|A\left(R_{\rho, M}^{A, \eta}\left(A\left(x_{1}\right)-\rho F\left(A\left(x_{1}\right)\right)\right)\right)-A\left(R_{\rho, M}^{A, \eta}\left(A\left(x_{2}\right)-\rho F\left(A\left(x_{2}\right)\right)\right)\right)\right\|^{q} \\
\leq & c_{q}\left\|A\left(x_{1}\right)-A\left(x_{2}\right)\right\|^{q} \\
& -q r\left\|A\left(R_{\rho, M}^{A, \eta}\left(A\left(x_{1}\right)-\rho F\left(A\left(x_{1}\right)\right)\right)\right)-A\left(R_{\rho, M}^{A, \eta}\left(A\left(x_{2}\right)-\rho F\left(A\left(x_{2}\right)\right)\right)\right)\right\|^{q} \\
& +\left\|A\left(R_{\rho, M}^{A, \eta}\left(A\left(x_{1}\right)-\rho F\left(A\left(x_{1}\right)\right)\right)\right)-A\left(R_{\rho, M}^{A, \eta}\left(A\left(x_{2}\right)-\rho F\left(A\left(x_{2}\right)\right)\right)\right)\right\|^{q} \\
\leq & c_{q}\left\|A\left(x_{1}\right)-A\left(x_{2}\right)\right\|^{q} \\
& -(q r-1)\left\|A\left(R_{\rho, M}^{A, \eta}\left(A\left(x_{1}\right)-\rho F\left(A\left(x_{1}\right)\right)\right)\right)-A\left(R_{\rho, M}^{A, \eta}\left(A\left(x_{2}\right)-\rho F\left(A\left(x_{2}\right)\right)\right)\right)\right\|^{q} .
\end{aligned}
$$

Therefore, (3.3) holds.

Lemma 3.3. Let $X$ be a q-uniformly smooth Banach space, $\eta: X \times X \rightarrow X$ be a $\tau$-Lipschtiz continuous mapping, $A: X \rightarrow X$ be an $r$-strongly $\eta$-accretive and nonexpansive mapping, $F: X \rightarrow X$ be an $\xi$-Lipschtiz continuous mapping, and $I_{k}=A-A R_{\rho, M}^{A, \eta}(A-\rho F A)$, and $M: X \rightarrow 2^{X}$ be an $(A, \eta)$-accretive set-valued mapping. Then the following statements are mutually equivalent.

(i) An element $x^{*} \in X$ is a solution of problem (2.1).

(ii) For a $x^{*} \in X$, such that

$$
x^{*}=R_{\rho, M}^{A, \eta}\left(A\left(x^{*}\right)-\rho F\left(A\left(x^{*}\right)\right)\right) .
$$

(iii) For a $x^{*} \in X$, holds

$$
I_{k}\left(x^{*}\right)=A\left(x^{*}\right)-A\left(R_{\rho, M}^{A, \eta}\left(A\left(x^{*}\right)-\rho F\left(A\left(x^{*}\right)\right)\right)\right)=0,
$$

where $\rho>0$ is a constant.

Proof. This directly follows from definitions of $R_{\rho, M(x)}^{A, \eta}$ and $I_{k}$.

Lemma 3.4. Let $X$ be a q-uniformly smooth Banach space, $\eta: X \times X \rightarrow X$ be a $\tau$-Lipschtiz continuous mapping, $A: X \rightarrow X$ be an $r$-strongly $\eta$-accretive and nonexpansive mapping, $F: X \rightarrow$ $X$ be an $\xi$-Lipschtiz continuous and $\beta$-strongly $\eta$-accretive mapping, and $I_{k}=A-A R_{\rho, M}^{A, \eta}(A-\rho F A)$, and $M: X \rightarrow 2^{X}$ be an $(A, \eta)$-accretive set-valued mapping. If the following conditions holds

$$
\tau^{q} \sqrt[q]{1+c_{q} \rho^{q} \xi^{q}-q \rho \beta}<\tau(r-m \rho) \quad\left(1+c_{q} \rho^{q} \xi^{q}>q \rho \beta\right)
$$


where $c_{q}>0$ is the same as in Lemma 2.5, and $\rho \in(0, r / m)$. Then the problem (2.1) has a solution $x^{*} \in X$.

Proof. Define $N: X \rightarrow X$ as follows:

$$
N(x)=R_{\rho, M}^{A, \eta}(A(x)-\rho F(A(x))), \quad \forall x \in X
$$

For elements $x_{1}, x_{2} \in X$, if letting

$$
s_{i}=A\left(x_{i}\right)-\rho F\left(A\left(x_{i}\right)\right) \quad(i=1,2)
$$

then by (3.1) and (3.3), we have

$$
\begin{aligned}
\left\|N\left(x_{1}\right)-N\left(x_{2}\right)\right\| & =\left\|R_{\rho, M}^{A, \eta}\left(s_{1}\right)-R_{\rho, M}^{A, \eta}\left(s_{2}\right)\right\| \\
& \leq \frac{\tau^{q-1}}{r-m \rho}\left(\left\|A\left(x_{1}\right)-A\left(x_{2}\right)-\rho\left(F\left(A\left(x_{1}\right)\right)-F\left(A\left(x_{2}\right)\right)\right)\right\|\right) .
\end{aligned}
$$

By using $r$-strongly $\eta$-accretive of $A, \beta$-strongly $\eta$-accretive of $F$, and Lemma 2.5 , we obtain

$$
\begin{aligned}
\| A\left(x_{1}\right) & -A\left(x_{2}\right)-\rho\left(F\left(A\left(x_{1}\right)\right)-F\left(A\left(x_{2}\right)\right)\right) \|^{q} \\
\leq & \left\|A\left(x_{1}\right)-A\left(x_{2}\right)\right\|^{q}+c_{q} \rho^{q}\left\|F\left(A\left(x_{1}\right)\right)-F\left(A\left(x_{2}\right)\right)\right\|^{q} \\
& -q \rho\left\langle F\left(A\left(x_{1}\right)\right)-F\left(A\left(x_{2}\right)\right), J_{q}\left(A\left(x_{1}\right)-A\left(x_{2}\right)\right)\right\rangle \\
\leq & \left(1+c_{q} \rho^{q} \xi^{q}-q \rho \beta\right)\left\|A\left(x_{1}\right)-A\left(x_{2}\right)\right\|^{q} .
\end{aligned}
$$

Combining (3.10)-(3.11), by using nonexpansivity of $A$, we have

$$
\left\|N\left(x_{1}\right)-N\left(x_{2}\right)\right\| \leq \theta^{*}\left\|x_{1}-x_{2}\right\|
$$

where

$$
\theta^{*}=\frac{\tau^{q-1}}{r-m \rho} \sqrt[q]{1+c_{q} \rho^{q} \xi^{q}-q \rho \beta} \quad\left(1+c_{q} \rho^{q} \xi^{q}>q \rho \beta\right)
$$

It follows from (3.7)-(3.12) that $N$ has a fixed point in $X$, that is, there exist a point $x^{*} \in X$ such that $x^{*}=N\left(x^{*}\right)$, and

$$
x^{*}=N\left(x^{*}\right)=R_{\rho, M}^{A, \eta}\left(A\left(x^{*}\right)-\rho F\left(A\left(x^{*}\right)\right)\right) .
$$

This completes the proof. 
Based on Lemma 3.3, we can develop a general over-relaxed $(A, \eta)$-proximal point algorithm to approximating solution of problem (2.1) as follows.

Algorithm 3.5. Let $X$ be a $q$-uniformly smooth Banach space, $\eta: X \times X \rightarrow X$ be a $\tau$-Lipschtiz continuous mapping, $A: X \rightarrow X$ be an $r$-strongly $\eta$-accretive and nonexpansive mapping, $F: X \rightarrow X$ be an $\beta$-strongly $\eta$-accretive mapping and $\xi$-Lipschitz continuous, and $I_{k}=$ $A-A R_{\rho, M}^{A, \eta}(A-\rho F A)$, and $M: X \rightarrow 2^{X}$ be an $(A, \eta)$-accretive set-valued mapping. Let $\left\{a_{n}\right\}_{n=0}^{\infty}\left(a_{n} \geq 1\right),\left\{b_{n}\right\}_{n=0}^{\infty}$ and $\left\{\rho_{n}\right\}_{n=0}^{\infty}$ be three nonnegative sequences such that

$$
\sum_{n=1}^{\infty} b_{n}<\infty, \quad a=\limsup _{n \rightarrow \infty} a_{n} \geq 1, \quad \rho_{n} \uparrow \rho \leq \infty,
$$

where $\rho_{n}, \rho \in(0, r / m)(n=0,1,2, \cdot, \cdot, \cdot)$ and each satisfies condition (3.7).

Step 1 . For an arbitrarily chosen initial point $x_{0} \in X$, set

$$
A\left(x_{1}\right)=\left(1-a_{0}\right) A\left(x_{0}\right)+a_{0} y_{0}
$$

where the $y_{0}$ satisfies

$$
\left\|y_{0}-A\left(R_{\rho_{0}, M}^{A, \eta}\left(A\left(x_{0}\right)-\rho_{0} F\left(A\left(x_{0}\right)\right)\right)\right)\right\| \leq b_{0}\left\|y_{0}-A\left(x_{0}\right)\right\| .
$$

Step 2. The sequence $\left\{x_{n}\right\}$ is generated by an iterative procedure

$$
A\left(x_{n+1}\right)=\left(1-a_{n}\right) A\left(x_{n}\right)+a_{n} y_{n}
$$

and $y_{n}$ satisfies

$$
\left\|y_{n}-A\left(R_{\rho_{n}, M}^{A, \eta}\left(A\left(x_{n}\right)-\rho_{n} F\left(A\left(x_{n}\right)\right)\right)\right)\right\| \leq b_{n}\left\|y_{n}-A\left(x_{n}\right)\right\|,
$$

where $n=1,2, \cdot, \cdot, \cdot \cdot$

Remark 3.6. For a suitable choice of the mappings $A, \eta, F, M, I_{k}$, and space $X$, then the Algorithm 3.5 can be degenerated to the hybrid proximal point algorithm $[16,17]$ and the over-relaxed $A$-proximal point algorithm [8].

Theorem 3.7. Let $X$ be a q-uniformly smooth Banach space. Let $A, F: X \rightarrow X$ and $\eta: X \times X \rightarrow X$ be single-valued mappings, and let $M: X \times X \rightarrow 2^{X}$ be a set-valued mapping and $(F A+M)^{-1}$ be the inverse mapping of the mapping $(F A+M): X \rightarrow 2^{X}$ satisfying the following conditions:

(i) $\eta: X \times X \rightarrow X$ is $\tau$-Lipschtiz continuous;

(ii) $A: X \rightarrow X$ be an $r$-strongly $\eta$-accretive mapping and nonexpansive;

(iii) $F: X \rightarrow X$ be an $\xi$-Lipschtiz continuous and $\beta$-strongly $\eta$-accretive mapping;

(iv) $M: X \rightarrow 2^{X}$ be an $(A, \eta)$-accretive set-valued mapping; 
(v) the $(F A+M)^{-1}$ be $(u, t)$-Lipschitz continuous at $0(u \geq 0)$;

(vi) $\left\{a_{n}\right\}_{n=0}^{\infty}\left(a_{n} \geq 1\right),\left\{b_{n}\right\}_{n=0}^{\infty}$ and $\left\{\rho_{n}\right\}_{n=0}^{\infty}$ be three nonnegative sequences such that

$$
\sum_{n=1}^{\infty} b_{n}<\infty, \quad a=\limsup _{n \rightarrow \infty} a_{n} \geq 1, \quad \rho_{n} \uparrow \rho \leq \infty,
$$

where $\rho_{n}, \rho \in(0, r / m)(n=0,1,2, \cdot, \cdot, \cdot)$ and each satisfies condition (3.7),

(vii) let the sequence $\left\{x_{n}\right\}$ generated by the general over-relaxed A-proximal point algorithm (3.6) be bounded and $x^{*}$ be a solution of problem (2.1), and the condition

$$
\begin{gathered}
\left\langle A\left(x_{n}\right)-A\left(x^{*}\right), J_{q}\left(A\left(R_{\rho, M}^{A, \eta}\left(A\left(x_{n}\right)-\rho F\left(A\left(x_{n}\right)\right)\right)\right)-A\left(R_{\rho, M}^{A, \eta}\left(A\left(x^{*}\right)-\rho F\left(A\left(x^{*}\right)\right)\right)\right)\right)\right\rangle \\
\geq \gamma\left\|A\left(R_{\rho, M}^{A, \eta}\left(A\left(x_{n}\right)-\rho F\left(A\left(x_{n}\right)\right)\right)\right)-A\left(R_{\rho, M}^{A, \eta}\left(A\left(x^{*}\right)-\rho F\left(A\left(x^{*}\right)\right)\right)\right)\right\|^{q} \\
0<c_{q}(a-1)^{q}+\left(a^{q}-q(a-1) a \gamma\right) d^{q}<1
\end{gathered}
$$

hold. Then the sequence $\left\{x_{n}\right\}$ converges linearly to a solution $x^{*}$ of problem (2.1) with convergence rate $\vartheta$, where

$$
\begin{gathered}
\vartheta=\sqrt[q]{c_{q}(a-1)^{q}+\left(a^{q}+q(1-a) a r\right) d^{q}}, \\
a=\limsup _{n \rightarrow \infty} a_{n}, \quad d=\limsup _{n \rightarrow \infty} d_{n}=\limsup _{n \rightarrow \infty} \sqrt[q]{\frac{c_{q} u^{q}}{(q r-1) r^{q} u^{q}+\rho_{n}^{q}}}, \quad \sum_{n=1}^{\infty} b_{n}<\infty .
\end{gathered}
$$

Proof. Let the $x^{*}$ be a solution of the Framework (2.1) for the conditions (i)-(iv) and Lemma 3.4. Suppose that the sequence $\left\{x_{n}\right\}$ which generated by the hybrid proximal point Algorithm 3.5 is bounded, from Lemma 3.4, we have

$$
A\left(x^{*}\right)=\left(1-a_{n}\right) A\left(x^{*}\right)+a_{n} A\left(R_{\rho_{n}, M}^{A, \eta}\left(A\left(x^{*}\right)-\rho_{n} F\left(A\left(x^{*}\right)\right)\right)\right) .
$$

We infer from Lemma 3.3 that any solution to (2.1) is a fixed point of $R_{\rho_{n}, M}^{A, \eta}\left(A-\rho_{n} F A\right)$. First, in the light of Lemma 3.2, we show

$$
\left\|R_{\rho_{n}, M}^{A, \eta}\left(A\left(x_{n}\right)-\rho_{n} F\left(A\left(x_{n}\right)\right)\right)-x^{*}\right\| \leq d_{n}\left\|A\left(x_{n}\right)-A\left(x^{*}\right)\right\|,
$$

where $d_{n}=\sqrt[q]{c_{q} u^{q} /\left((2 \gamma-1) r^{q} u^{q}+\rho_{n}^{q}\right)}<1$ and $R_{\rho_{n}, M}^{A, \eta}\left(A\left(x^{*}\right)-\rho_{n} F\left(A\left(x^{*}\right)\right)\right)=x^{*}$. 
For $I_{k}=A-A R_{\rho, M}^{A, \eta}\left(A-\rho_{n} F A\right)$, and under the assumptions (including the condition (vii) (3.21)), then $I_{k}\left(x_{n}\right) \rightarrow 0(n \rightarrow \infty)$ since the $(F A+M)^{-1}$ is $(u, t)$-Lipschitz continuous at 0 . Indeed, it follows that $R_{\rho_{n}, M}^{A, \eta}\left(A\left(x_{n}\right)-\rho_{n} F\left(A\left(x_{n}\right)\right)\right) \in(F A+M)^{-1}\left(\rho_{n}^{-1} I_{k}\left(x_{n}\right)\right)$ from $\rho_{n}^{-1} I_{k}\left(x_{n}\right) \in$ $(F A+M)\left(R_{\rho_{n}, M}^{A, \eta}\left(A\left(x_{n}\right)-\rho_{n} F\left(A\left(x_{n}\right)\right)\right)\right)$. Next, by using the condition (iv) and (3.1), and setting $w=\rho_{n}^{-1} I_{k}\left(x_{n}\right)$ and $z=R_{\rho_{n}, M}^{A, \eta}\left(A\left(x_{n}\right)-\rho_{n} F\left(A\left(x_{n}\right)\right)\right)$, we have

$$
\left\|R_{\rho_{n}, M}^{A, \eta}\left(A\left(x_{n}\right)-\rho_{n} F\left(A\left(x_{n}\right)\right)\right)-x^{*}\right\| \leq u\left\|\rho_{n}^{-1} I_{k}\left(x_{n}\right)\right\|, \quad \forall n>n^{\prime} .
$$

Now applying Lemma 3.3, we get

$$
\begin{aligned}
& \left\|R_{\rho_{n}, M}^{A, \eta}\left(A\left(x_{n}\right)-\rho_{n} F\left(A\left(x_{n}\right)\right)\right)-x^{*}\right\|^{q} \\
& \leq\left\|R_{\rho_{n}, M}^{A, \eta}\left(A\left(x_{n}\right)-\rho_{n} F\left(A\left(x_{n}\right)\right)\right)-R_{\rho_{n}, M}^{A, \eta}\left(A\left(x^{*}\right)-\rho_{n} F\left(A\left(x^{*}\right)\right)\right)\right\|^{q} \\
& \leq u^{q}\left\|\rho_{n}^{-1} I_{k}\left(x_{n}\right)-\rho_{n}^{-1} I_{k}\left(x^{*}\right)\right\|^{q} \\
& \leq\left(\frac{u}{\rho_{n}}\right)^{q}\left\|I_{k}\left(x_{n}\right)-I_{k}\left(x^{*}\right)\right\|^{q} \\
& \leq\left(\frac{u}{\rho_{n}}\right)^{q}\left[-(q r-1) r^{q}\left\|R_{\rho_{n}, M}^{A, \eta}\left(A\left(x_{n}\right)-\rho_{n} F\left(A\left(x_{n}\right)\right)\right)-R_{\rho_{n}, M}^{A, \eta}\left(A\left(x^{*}\right)-\rho_{n} F\left(A\left(x^{*}\right)\right)\right)\right\|^{q}\right. \\
& \left.\quad+c_{q}\left\|A\left(x_{n}\right)-A\left(x^{*}\right)\right\|^{q}\right] .
\end{aligned}
$$

Therefore,

$$
\left\|R_{\rho_{n}, M}^{A, \eta}\left(A\left(x_{n}\right)-\rho_{n} F\left(A\left(x_{n}\right)\right)\right)-x^{*}\right\| \leq d_{n}\left\|A\left(x_{n}\right)-A\left(x^{*}\right)\right\|,
$$

where $d_{n}=\sqrt[q]{c_{q} u^{q} /\left((2 \gamma-1) r^{q} u^{q}+\rho_{n}^{q}\right)}<1$ and $R_{\rho_{n}, M}^{A, \eta}\left(A\left(x^{*}\right)-\rho_{n} F\left(A\left(x^{*}\right)\right)\right)=x^{*}$.

Next we start the main part of the proof by using the expression

$$
A\left(z_{n+1}\right)=\left(1-a_{n}\right) A\left(x_{n}\right)+a_{n} A\left(R_{\rho_{n}, M}^{A, \eta}\left(A\left(x_{n}\right)-\rho_{n} F\left(A\left(x_{n}\right)\right)\right)\right), \quad \forall n \geq 0 .
$$


Let us set $s_{n}=A\left(x_{n}\right)-\rho_{n} F\left(A\left(x_{n}\right)\right)$ and $s^{*}=A\left(x^{*}\right)-\rho_{n} F\left(A\left(x^{*}\right)\right)$ for simple. We begin with estimating (for $a_{n} \geq 1$ ) and later using (3.2), the nonexpansivity of $A,(3.21)$ and (3.28) as follows:

$$
\begin{aligned}
\left\|A\left(z_{n+1}\right)-A\left(x^{*}\right)\right\|^{q} \leq & \|\left(1-a_{n}\right) A\left(x_{n}\right)+a_{n} A\left(R_{\rho_{n}, M}^{A, \eta}\left(s_{n}\right)\right) \\
& -\left[\left(1-a_{n}\right) A\left(x^{*}\right)+a_{n} A\left(R_{\rho_{n}, M}^{A, \eta}\left(s^{*}\right)\right)\right] \|^{q} \\
\leq & \left\|\left(1-a_{n}\right)\left(A\left(x_{n}\right)-A\left(x^{*}\right)\right)+a_{n}\left[A\left(R_{\rho_{n}, M}^{A, \eta}\left(s_{n}\right)\right)-A\left(R_{\rho_{n}, M}^{A, \eta}\left(s^{*}\right)\right)\right]\right\|^{q} \\
\leq & c_{q}\left\|\left(1-a_{n}\right)\left(A\left(x_{n}\right)-A\left(x^{*}\right)\right)\right\|^{q}+\left\|a_{n}\left[A\left(R_{\rho_{n}, M}^{A, \eta}\left(s_{n}\right)\right)-A\left(R_{\rho_{n}, M}^{A, \eta}\left(s^{*}\right)\right)\right]\right\|^{q} \\
& +q\left(1-a_{n}\right) a_{n}\left\langle A\left(x_{n}\right)-A\left(x^{*}\right), J_{q}\left(A\left(R_{\rho_{n}, M}^{A, \eta}\left(s_{n}\right)\right)-A\left(R_{\rho_{n}, M}^{A, \eta}\left(s^{*}\right)\right)\right)\right\rangle \\
\leq & c_{q}\left(a_{n}-1\right)^{q}\left\|A\left(x_{n}\right)-A\left(x^{*}\right)\right\|^{q}+a_{n}^{q} \|\left(R_{\rho_{n}, M}^{A, \eta}\left(s_{n}\right)-R_{\rho_{n}, M}^{A, \eta}\left(s^{*}\right) \|^{q}\right. \\
& +q\left(1-a_{n}\right) a_{n} \gamma \|\left(R_{\rho_{n}, M}^{A, \eta}\left(s_{n}\right)-R_{\rho_{n}, M}^{A, \eta}\left(s^{*}\right) \|^{q}\right. \\
\leq & c_{q}\left(a_{n}-1\right)^{q}\left\|A\left(x_{n}\right)-A\left(x^{*}\right)\right\|^{q} \\
& +\left[a_{n}^{q}-q\left(1-a_{n}\right) a_{n} \gamma\right] \|\left(R_{\rho_{n}, M}^{A, \eta}\left(A\left(x_{n}\right)-\rho_{n} F\left(A\left(x_{n}\right)\right)\right)-x^{*} \|^{q}\right. \\
\leq & {\left[c_{q}\left(a_{n}-1\right)^{q}+\left(a_{n}^{q}+q\left(1-a_{n}\right) a_{n} \gamma\right) d_{n}^{q}\right]\left\|A\left(x_{n}\right)-A\left(x^{*}\right)\right\|^{q} . }
\end{aligned}
$$

Thus, we have

$$
\left\|A\left(z_{n+1}\right)-A\left(x^{*}\right)\right\|^{q} \leq \theta_{n}\left\|A\left(x_{n}\right)-A\left(x^{*}\right)\right\|^{q},
$$

where

$$
\theta_{n}=\sqrt[q]{c_{q}\left(a_{n}-1\right)^{q}+\left(a_{n}^{q}+q\left(1-a_{n}\right) a_{n} \gamma\right) d_{n}^{q}}<1
$$

and $a_{n}^{q}+q\left(1-a_{n}\right) a_{n} \gamma>0, a_{n} \geq 1, \sum_{n=1}^{\infty} b_{n}<\infty$, and $d_{n}=\sqrt[q]{c_{q} u^{q} /\left((2 \gamma-1) r^{q} u^{q}+\rho_{n}^{q}\right)}<1$.

Since $A\left(x_{n+1}\right)=\left(1-a_{n}\right) A\left(x_{n}\right)+a_{n} y_{n}$, we have $A\left(x_{n+1}\right)-A\left(x_{n}\right)=a_{n}\left(y_{n}-A\left(x_{n}\right)\right)$. It follows that

$$
\begin{aligned}
& \left\|A\left(x_{n+1}\right)-A\left(z_{n+1}\right)\right\| \\
& \quad \leq\left\|\left(1-a_{n}\right) A\left(x_{n}\right)+a_{n} y_{n}-\left[\left(1-a_{n}\right) A\left(x_{n}\right)+a_{n} R_{\rho_{n}, M}^{A, \eta}\left(A\left(x_{n}\right)-\rho_{n} F\left(A\left(x_{n}\right)\right)\right)\right]\right\| \\
& \quad \leq a_{n}\left\|y_{n}-R_{\rho_{n}, M}^{A, \eta}\left(A\left(x_{n}\right)-\rho_{n} F\left(A\left(x_{n}\right)\right)\right)\right\| \\
& \quad \leq a_{n} b_{n}\left\|y_{n}-A\left(x_{n}\right)\right\| .
\end{aligned}
$$


Next, we can obtain

$$
\begin{aligned}
\left\|A\left(x_{n+1}\right)-A\left(x^{*}\right)\right\| & \leq\left\|A\left(z_{n+1}\right)-A\left(x^{*}\right)\right\|+\left\|A\left(x_{n+1}\right)-A\left(z_{n+1}\right)\right\| \\
& \leq\left\|A\left(z_{n+1}\right)-A\left(x^{*}\right)\right\|+a_{n} b_{n}\left\|y_{n}-A\left(x_{n}\right)\right\| \\
& \leq\left\|A\left(z_{n+1}\right)-A\left(x^{*}\right)\right\|+b_{n}\left\|A\left(x_{n+1}\right)-A\left(x_{n}\right)\right\| \\
& \leq\left\|A\left(z_{n+1}\right)-A\left(x^{*}\right)\right\|+b_{n}\left\|A\left(x_{n+1}\right)-A\left(x^{*}\right)\right\|+b_{n}\left\|A\left(x^{*}\right)-A\left(x_{n}\right)\right\| .
\end{aligned}
$$

This implies from (3.38) and (3.39) that

$$
\left\|A\left(x_{n+1}\right)-A\left(x^{*}\right)\right\| \leq \frac{\theta_{n}+b_{n}}{1-b_{n}}\left\|A\left(x_{n}\right)-A\left(x^{*}\right)\right\|
$$

Since $A$ is an $r$-strongly $\eta$-accretive mapping (and hence, $\|A(x)-A(y)\| \geq r\|x-y\|$ ), this implies from (3.35) that the sequence $\left\{x_{n}\right\}$ converges strongly to $x^{*}$ for

$$
\theta_{n}=\sqrt[q]{c_{q}\left(a_{n}-1\right)^{q}+\left(a_{n}^{q}+q\left(1-a_{n}\right) a_{n} \gamma\right) d_{n}^{q}}<1,
$$

where $a_{n} \geq 1, \sum_{n=1}^{\infty} b_{n}<\infty$, and $d_{n}=\sqrt[q]{c_{q} u^{q} /\left((2 \gamma-1) r^{q} u^{q}+\rho_{n}^{q}\right)}<1$.

Hence, we have

$$
\begin{aligned}
\vartheta & =\limsup _{n \rightarrow \infty} \frac{\theta_{n}+b_{n}}{1-b_{n}}=\limsup _{n \rightarrow \infty} \theta_{n} \\
& =\sqrt[q]{c_{q}(a-1)^{q}+\left(a^{q}+q(1-a) a \gamma\right) d^{q}} .
\end{aligned}
$$

By (3.22), it follows that $0<\vartheta<1$ from the condition (vi), and the sequence $\left\{x_{n}\right\}$ generated by the hybrid proximal point Algorithm 3.5 converges linearly to a solution $x^{*}$ of problem (2.1) with convergence rate $\vartheta$. This completes the proof.

Corollary 3.8. Let $X$ be a Hilbert space $\left(q=2, c_{q}=1\right), A: X \rightarrow X$ be an $r$-strongly monotone and nonexpansive mapping $(\alpha=1), F=0$ is a zero operator, $M: X \rightarrow 2^{X}$ be an A-maximal setvalued monotone. $I_{k}=A-A R_{\rho, M}^{A, \eta}\left(A-\rho_{n} F A\right)$, and the condition (3.21) hold, the $M^{-1}$ be u-Lipschitz continuous at $0(u \geq 0)$. Let $\left\{a_{n}\right\}_{n=0}^{\infty},\left\{b_{n}\right\}_{n=0}^{\infty}$ and $\left\{\rho_{n}\right\}_{n=0}^{\infty}$ be the same as in Algorithm 3.5. If

$$
0<1-a\left[2\left(1-\gamma d^{2}\right)-a\left(1-(2 \gamma-1) d^{2}\right)\right]<1,
$$

then the bounded sequence $\left\{x_{n}\right\}$ generated by the general over-relaxed A-proximal point algorithm converges linearly to a solution $x^{*}$ of problem (2.1) with convergence rate $\vartheta$, where

$$
\vartheta=\sqrt{1-a\left[2\left(1-\gamma d^{2}\right)-a\left(1-(2 \gamma-1) d^{2}\right)\right]},
$$


and $d=\limsup _{n \rightarrow \infty} d_{n}=\limsup _{n \rightarrow \infty} \sqrt{u^{2} /\left((2 \gamma-1) r^{2} u^{2}+\rho_{n}^{2}\right)}=\sqrt{u^{2} /\left((2 \gamma-1) r^{2} u^{2}+\rho^{2}\right)}, a=$ $\limsup _{n \rightarrow \infty} a_{n}, \sum_{n=1}^{\infty} b_{n}<\infty$.

This is Theorem 3.2 in [8], and if, in addition, $\gamma=1, A=I$ then we can have the Proposition 2 in [9].

\section{References}

[1] X. P. Ding and C. L. Luo, "Perturbed proximal point algorithms for general quasi-variational-like inclusions," Journal of Computational and Applied Mathematics, vol. 113, no. 1-2, pp. 153-165, 2000.

[2] R. U. Verma, "Approximation-solvability of a class of A-monotone variational inclusion problems," Journal KSIAM, vol. 8, no. 1, pp. 55-66, 2004.

[3] N.-J. Huang, "Nonlinear implicit quasi-variational inclusions involving generalized $m$-accretive mappings," Archives of Inequalities and Applications, vol. 2, no. 4, pp. 413-425, 2004.

[4] Y.-P. Fang and N.-J. Huang, "H-accretive operators and resolvent operator technique for solving variational inclusions in Banach spaces," Applied Mathematics Letters, vol. 17, no. 6, pp. 647-653, 2004.

[5] Y.-P. Fang, N.-J. Huang, and H. B. Thompson, "A new system of variational inclusions with $(H, \eta)$ monotone operators in Hilbert spaces," Computers \& Mathematics with Applications, vol. 49, no. 2-3, pp. 365-374, 2005.

[6] H.-Y. Lan, Y. J. Cho, and R. U. Verma, "Nonlinear relaxed cocoercive variational inclusions involving $(A, \eta)$-accretive mappings in Banach spaces," Computers $\mathcal{E}$ Mathematics with Applications, vol. 51, no. 9-10, pp. 1529-1538, 2006.

[7] Q.-B. Zhang, X.-P. Ding, and C.-Z. Cheng, "Resolvent operator technique for generalized implicit variational-like inclusion in Banach space," Journal of Mathematical Analysis and Applications, vol. 361, no. 2, pp. 283-292, 2010.

[8] R. U. Verma, “A general framework for the over-relaxed $A$-proximal point algorithm and applications to inclusion problems," Applied Mathematics Letters, vol. 22, no. 5, pp. 698-703, 2009.

[9] T. Pennanen, "Local convergence of the proximal point algorithm and multiplier methods without monotonicity," Mathematics of Operations Research, vol. 27, no. 1, pp. 170-191, 2002.

[10] H.-G. Li, "Iterative algorithm for a new class of generalized nonlinear fuzzy set-variational inclusions involving $(H, \eta)$-monotone mappings," Advances in Nonlinear Variational Inequalities, vol. 10, no. 1, pp. 89-100, 2007.

[11] H. G. Li, "Approximate algorithm of solutions for general nonlinear fuzzy multivalued quasivariational inclusions with $(G, \eta)$-monotone mappings," Nonlinear Functional Analysis and Applications, vol. 13, no. 2, pp. 277-289, 2008.

[12] H.-G. Li, "Perturbed Ishikawa iterative algorithm and stability for nonlinear mixed quasi-variational inclusions involving $(A, \eta)$-accretive mappings," Advances in Nonlinear Variational Inequalities, vol. 11, no. 1, pp. 41-50, 2008.

[13] H.-Y. Lan, "On multivalued nonlinear variational inclusion problems with $(A, \eta)$-accretive mappings in Banach spaces," Journal of Inequalities and Applications, vol. 2006, Article ID 59836, 12 pages, 2006.

[14] H. K. Xu, "Inequalities in Banach spaces with applications," Nonlinear Analysis: Theory, Methods $\mathcal{E}$ Applications, vol. 16, no. 12, pp. 1127-1138, 1991.

[15] X. Weng, "Fixed point iteration for local strictly pseudo-contractive mapping," Proceedings of the American Mathematical Society, vol. 113, no. 3, pp. 727-731, 1991.

[16] R. U. Verma, "A hybrid proximal point algorithm based on the $(A, \eta)$-maximal monotonicity framework," Applied Mathematics Letters, vol. 21, no. 2, pp. 142-147, 2008.

[17] H. G. Li, A. J. Xu, and M. M. Jin, "An Ishikawa-hybrid proximal point algorithm for nonlinear setvalued inclusions problem based on $(A, \eta)$-accretive framework," Fixed Point Theory and Applications, vol. 2010, Article ID 501293, 12 pages, 2010. 